\title{
Oxidative phosphorylation as an emerging target in cancer therapy
}

\section{Running Title: OXPHOS as an emerging target in cancer therapy}

Authors: Thomas M. Ashton ${ }^{\mathrm{a}}$, W. Gillies McKenna ${ }^{\mathrm{a}}$, Leoni A. Kunz-Schughart ${ }^{\mathrm{a}, \mathrm{b}, \mathrm{c}, *}$, Geoff S. Higgins ${ }^{\mathrm{a},}$.

\section{Author Affiliations:}

${ }^{\mathrm{a} C R U K / M R C ~ O x f o r d ~ I n s t i t u t e ~ f o r ~ R a d i a t i o n ~ O n c o l o g y, ~ G r a y ~ L a b o r a t o r i e s, ~ O l d ~ R o a d ~ C a m p u s ~}$ Research Building, Roosevelt Drive, Oxford, OX3 7DQ.

${ }^{\mathrm{b}}$ OncoRay - National Center for Radiation Research in Oncology, Faculty of Medicine and University Hospital Carl Gustav Carus, TU Dresden, and Helmholtz-Zentrum Dresden Rossendorf, Germany

${ }^{\mathrm{c}}$ National Center for Tumor Diseases (NCT), partner site Dresden, Germany

*These authors contributed equally to this work

\section{Corresponding Authors:}

Geoff S. Higgins, CRUK/MRC Oxford Institute for Radiation Oncology, Gray Laboratories, Old Road Campus Research Building, Roosevelt Drive, Oxford, OX3 7DQ; Phone: +44 (0)1865 617311; Email: geoffrey.higgins@ oncology.ox.ac.uk

Leoni A. Kunz-Schughart, OncoRay-National Center for Radiation Research in Oncology, Faculty of Medicine and University Hospital Carl Gustav Carus, TU Dresden, and HelmholtzZentrum Dresden - Rossendorf, Institute of Radiooncology, Germany; Phone: +49 (0)351 458 7405; Email: leoni.kunz-schughart@ oncoray.de

Email of other authors:

thomas.ashton@oncology.ox.ac.uk; gillies.mckenna@oncology.ox.ac.uk 
Conflict of interest: The authors declare no competing interests.

Financial Support: The funding sources were Cancer Research UK (Higgins C34326/A13092, McKenna C5255/A23755) and the Medical Research Council (McKenna MC_PC_12004).

\title{
Oxidative phosphorylation as an emerging target in cancer therapy
}

\begin{abstract}
Cancer cells have upregulated glycolysis compared to normal cells, which has led many to the assumption that oxidative phosphorylation (OXPHOS) is downregulated in all cancers. However, recent studies have shown that OXPHOS can be also upregulated in certain cancers, including leukemias, lymphomas, pancreatic ductal adenocarcinoma, high OXPHOS subtype melanoma and endometrial carcinoma, and that this can occur even in the face of active glycolysis. OXPHOS inhibitors could therefore be used to target cancer subtypes in which OXPHOS is upregulated, and to alleviate therapeutically adverse tumor hypoxia. Several drugs including metformin, atovaquone and arsenic trioxide are used clinically for non-oncological indications, but emerging data demonstrates their potential use as OXPHOS inhibitors. We highlight novel applications of OXPHOS inhibitors with a suitable therapeutic index to target cancer cell metabolism.
\end{abstract}

\section{Keywords}

Oxidative phosphorylation; electron transport; cancer metabolism; mitochondria; Tumor hypoxia. 


\section{Introduction}

In the 1920s, Otto Warburg discovered that even well oxygenated cancer cells have high glucose consumption and high lactate production, indicating that glycolysis is upregulated. The observation that cancer cells have upregulated glycolysis compared to normal cells lead to the assumption that oxidative phosphorylation (OXPHOS) is universally downregulated in cancer. This is indeed the case for many cancers, but in some cancers this assumption is being challenged by an increasing body of evidence to suggest that mitochondrial metabolism is not impaired, including leukemias, lymphomas, pancreatic ductal adenocarcinoma, high OXPHOS subtype melanoma and endometrial carcinoma $(1,2)$.

The oxidative phosphorylation (OXPHOS) metabolic pathway generates ATP by transport of electrons to a series of transmembrane protein complexes in the mitochondrial inner membrane, known as the electron transport chain (ETC). NADH, $\mathrm{FADH}_{2}$ and succinate act as electron donors. As the electrons pass through the multi-protein ETC complexes I - IV, protons are pumped from the mitochondrial matrix into the intermembrane space by complexes I, III and IV (Fig. 1). When OXPHOS is active there is a high proton gradient across the membrane, and protons flow from the inner inter-membrane space back into the mitochondrial matrix through complex V, ATP synthase, driving the synthesis of ATP. Oxygen acts as the terminal electron acceptor.

The last five years have heralded novel uses for OXPHOS inhibitors either to treat cancers in which OXPHOS is upregulated or to alleviate tumor hypoxia in order to improve treatment outcomes. Alleviation of tumor hypoxia may be achieved in cancers in which OXPHOS is not upregulated, so this approach could be widely applicable. Several recent reviews have highlighted mitochondrial metabolism as a target for anti-cancer therapy, with a particular focus on metformin as an OXPHOS inhibitor (1,3-9). This review discusses novel applications of a wide range of OXPHOS inhibitors that have a suitable therapeutic index to target cancer cell metabolism. 


\section{OXPHOS as an anti-cancer target}

\section{Reduced OXPHOS activity in cancer}

There is a group of cancers in which OXPHOS is downregulated, and in those cancers decreased OXPHOS activity may be related to mitochondrial DNA (mtDNA) mutations, or reduced mtDNA content, as mtDNA codes for 13 subunits of OXPHOS protein complexes I, III, IV and V (10). OXPHOS downregulation is associated with poor clinical outcome across all cancer types, and correlates with a gene signature characteristic of invasive and metastatic tumors (11). Decreases in mtDNA content have been observed in a range of cancers including breast, gastric, hepatocellular carcinoma and non-small cell lung (NSCLC) cancers. However, in some cancers mtDNA is a requirement for tumorigenesis, for cancer cells to grow in an anchorage-dependent manner, and to mediate resistance to cytotoxic drugs $(9,12)$. For example, Weinberg et al. demonstrated that mitochondrial metabolism and ROS generation are essential for Kras-mediated tumorigenicity (13).

Mitochondrial genome sequence analysis of 226 paired tumor and normal tissue samples from The Cancer Genome Atlas (TCGA) revealed deleterious tumor-specific somatic mtDNA mutations in $63 \%$ of rectal adenocarcinomas, $53 \%$ of colon adenocarcinomas, $36 \%$ of ovarian serous cyst adenocarcinomas and $30 \%$ of acute myeloid leukemias (14). Mutations were identified in all mitochondrially-encoded genes, and are predicted to impact protein function, potentially affecting OXPHOS levels. Interestingly, however, cancer cells harboring mtDNA mutations in complex I subunits were 5 to 20 fold more sensitive to the complex I inhibitors, metformin and phenformin, compared to cell lines lacking such mutations (15). Metformin is a biguanide widely used to treat type-2 diabetes, and phenformin is a precursor of metformin not currently in clinical use. Phenformin also inhibited the growth of xenografts derived from two independent cell lines (Cal-62 and U937) harboring mtDNA mutations. This study thus demonstrated that complex I inhibition causes decreased growth in cells with mtDNA mutations in complex I subunits. It is also 
important to note that many mtDNA mutations do not simply cause a decrease in OXPHOS, but may facilitate adaptation to the bioenergetic demands of the tumor microenvironment without altering OXPHOS (6).

\section{OXPHOS is upregulated in some cancers}

There is an increasing body of evidence demonstrating that certain cancers are heavily reliant on OXPHOS, and many recent studies have revealed that OXPHOS inhibition is effective in targeting these cancer subtypes (Table 1). A meta-analysis of 16 normal cell types and 31 cancer cell lines indicated that the relative contribution of glycolysis and OXPHOS to ATP production is highly variable between cell types, but that the average contribution of OXPHOS to ATP production is $80 \%$ in normal cells and $83 \%$ in cancer cells (16). This is in accordance with the in vivo data from Vaupel's group demonstrating that the availability of $\mathrm{O}_{2}$ in solid tumors is the key determinant of the oxygen consumption rate (OCR), suggesting that mitochondrial respiratory capacity is not always functionally impaired (17). One cause of the variability in the contribution of OXPHOS between cancer types may be mtDNA content. Many cancers have increased mtDNA content relative to normal tissue, including acute lymphoblastic leukemia (ALL), Non-Hodgkin lymphoma, endometrial, colorectal, ovarian, prostate, head and neck, lung adenocarcinoma, esophageal squamous cell carcinoma, and thyroid cancers $(10,18)$. To add further complexity, recent studies suggest that tumors may be metabolically heterogenous, and that cancer stem cells with high metastatic and tumorigenic potential are more reliant upon OXPHOS than the bulk, and putatively non-stem, component of pancreatic tumors $(9,19)$. Metabolic heterogeneity has also been demonstrated in NSCLC tumors $(20,21)$. As analysis of mtDNA content or the expression of OXPHOS genes may not reflect the level of functional OXPHOS, it is important to pursue a multi-experimental approach to fully characterize OXPHOS activity. Therefore examples are provided of tumor types in which high OXPHOS gene expression correlates with high OXPHOS protein levels as determined by IHC or proteomics, and high 
OXPHOS activity as determined by metabolomics, oxygen consumption or sensitivity to well characterized OXPHOS inhibitors.

Several studies indicate that OXPHOS may be upregulated in breast cancer and classical Hodgkin lymphoma. Complex I, II and IV activity respectively assayed by NADH, succinate dehydrogenase and cytochrome oxidase histochemical staining of breast cancer tissue reveals that ETC proteins are upregulated in breast cancer cells relative to adjacent stromal and normal epithelial cells (22). The activity of these complexes could be overcome by treatment of the tissue sections with metformin or sodium azide, an inhibitor of complex IV. Analysis of gene expression data from 2000 breast cancer patients revealed significant transcriptional upregulation of OXPHOS, suggesting that OXPHOS is a possible target in breast cancer (22). Transcriptomic data and western blotting demonstrated that OXPHOS is highly upregulated in breast cancers deficient in RB1, a protein lost in 20-30\% of basal-like breast cancers $(23,24)$. The mitochondrial translation inhibitor, tigecycline, strongly attenuated growth of $R B 1$-deficient MDA-MB-436 breast xenografts (24). OXPHOS is also globally upregulated in classical Hodgkin lymphoma, with an increase in expression of OXPHOS genes, increase in mitochondrial mass, increase in ETC protein expression, increase in oxygen consumption rate and decrease in lactate production promoted by $\mathrm{NF} \kappa \mathrm{B}$ (25). In many cancers, however, OXPHOS upregulation is limited to particular cancer subtypes as exemplified below.

Diffuse large B cell lymphomas (DLBCL) can be divided into OXPHOS high and low subsets (26). Mitochondrial proteomics and gene expression analysis revealed that ETC components are upregulated in the OXPHOS high subset, particularly subunits of complexes I and IV. OXPHOS is also enhanced in acute myeloid leukemia (AML) stem cells, dependent upon expression of the $B C L-2$ oncogene (27). Inhibition of BCL-2 reduces OXPHOS and selectively eradicates quiescent chemotherapy-resistant AML stem cells. Expression of genes other than BCL-2 also alters the reliance on OXPHOS, as AML cells with low basal 
phosphorylation of AKT or low basal glycolysis have increased OXPHOS and greater sensitivity to the complex I inhibitor, metformin, reducing leukemia growth in vivo (28).

Transcriptomic and metabolic analyses of Ras-driven pancreatic ductal adenocarcinoma (PDAC) stem-like cells reveal a strong reliance on OXPHOS and decreased glycolysis (19). These cells are highly resistant to conventional chemotherapies, and are able to repopulate heterogenous cancer cell populations (29). Treatment with metformin or the complex $\mathrm{V}$ inhibitor, oligomycin, retards growth of these cells in vitro and causes growth delay of PDAC-215 and PDAC-A6L xenografts (29). Furthermore, immortalization and transformation of bronchial epithelial cells with the H-Ras ${ }^{\mathrm{V} 12}$ oncogenic Ras allele causes an increase in the OCR, and expression of $\mathrm{H}-\mathrm{Ras}^{\mathrm{V} 12}$ increases sensitivity to the complex I inhibitor, rotenone (30). Therapy-resistant chronic myeloid leukemia stem cells also have upregulated OXPHOS, as determined by metabolomics and functional assays (31).

It is important to note that tumors can display metabolic flexibility $(5,7)$, so a high reliance on OXPHOS does not necessarily confer dependence. Tumors with a high reliance on OXPHOS that are able to switch to glycolysis for ATP production may still be susceptible to OXPHOS inhibition, but this remains to be determined experimentally.

\section{Molecularly targeted therapy can cause OXPHOS upregulation}

Several cases have been described in which cancer cells become more dependent upon OXPHOS following treatment with targeted therapies, including inhibition of the protein kinase, BRAF, in melanomas with an activating mutation in the $B R A F$ gene. Roughly $50 \%$ of melanomas carry activating BRAF mutations, such as BRAF V600E, and are therefore initially susceptible to BRAF inhibitors. BRAF inhibitors induce PGC1 $\alpha$, a regulator of mitochondrial biogenesis, which in turn causes OXPHOS dependence (32). Consequently, BRAF inhibitors synergize with the complex I inhibitor, phenformin, to reduce the viability of BRAF V600E mutant melanoma cells and to induce tumor regression in a $\mathrm{BRAF}^{\mathrm{V} 600 \mathrm{E}} / \mathrm{PTEN}^{\text {null }}$-driven mouse melanoma model (33). In addition, there is a subset of 
melanomas that have high PGC1 $\alpha$ expression and high levels of OXPHOS that does not appear to correlate with BRAF or p53 mutational status (34). OXPHOS inhibitors may thus be useful as standalone agents for the treatment of melanomas with high PGC1 $\alpha$ expression, and in combination with BRAF inhibitors for targeting BRAF mutant melanomas.

\section{OXPHOS upregulation can be driven by gene mutation}

The characterization of cancer cells with an OXPHOS phenotype and gene mutations driving OXPHOS upregulation is ongoing. For example, non-small lung cell cancer (NSCLC) tumors with oncogenic Kras and loss of the LKB1 tumor suppressor are selectively sensitive to the complex I inhibitor, phenformin (35). Phenformin and rotenone caused complete inhibition of oxygen consumption in these cells, demonstrating OXPHOS functionality. About $20 \%$ of all NSCLC have mutated LKB1. LKB1 is the primary kinase responsible for activation of AMPK, which is required to enhance glycolysis to compensate for the reduction in ATP under reduced OXPHOS. NSCLC tumors that are unable to sufficiently upregulate glycolysis are thus particularly sensitive to OXPHOS inhibition. AMPK-independent activation of stress signaling pathways is also considered to contribute to the sensitivity of these cells to phenformin.

\section{OCR inhibition to alleviate tumor hypoxia}

\section{Hypoxia is associated with poor clinical outcomes}

It has been known since the work of Gray and his colleagues in the 1950s that solid tumors frequently have regions of low oxygen known as hypoxia, which result from an imbalance between oxygen demand and poor oxygen supply due to abnormal vasculature (36,37). As Gray predicted, and has been frequently subsequently demonstrated, tumor hypoxia results in worse clinical outcomes because hypoxic cells are resistant to cancer therapy, leading to local recurrence and an increased propensity towards metastasis (38). 
Tumor hypoxia is known to be associated with poor clinical outcomes in many cancers including head and neck, cervix, lung, brain, bowel, prostate and pancreas (36). Hypoxic tumor cells are also up to three times more resistant to radiotherapy than normoxic tumor cells due to the absence of the oxygen enhancement effect (37). This effect is a result of the reactive oxygen species (ROS) generated by the radiolysis of water that attack DNA, forming readily reversible DNA radicals. These radicals are converted into DNA peroxides in the presence of oxygen, which must be physically present within microseconds of the damage, forming more stable intermediates that are more difficult to repair (39). Even very low levels of oxygen, around $2 \%$, are sufficient to yield oxygen enhancement.

\section{Strategies for the modification of tumor hypoxia}

Previous attempts to overcome tumor hypoxia have included the use of nitroimidazoles such as misonidazole and nimorazole, inhalation of hyperbaric oxygen, and the use of carbogen $\left(95 \% \mathrm{O}_{2}, 5 \% \mathrm{CO}_{2}\right)$ in combination with the vasodilator, nicotinamide (ARCON). The reasons why these attempts at increasing oxygen 'supply' have had limited clinical success are multifactorial. However, the use of drugs that were poorly tolerated, practical challenges associated with delivering some of these treatments, and the absence of predictive biomarkers, all contributed to the failure of these treatments to enter widespread clinical use. An additional drawback to approaches that require a drug to be delivered to hypoxic tumor regions is that these regions are usually poorly vascularized, so high doses may be required to achieve the local drug concentrations required to elicit an effect. A more novel approach is to reduce the OCR, increasing the retention of oxygen throughout the tumor and subsequently decreasing tumor hypoxia. This could be achieved with OXPHOS inhibition (Fig. 2), as shall be further highlighted (39-42). 
Reduction of oxygen consumption alleviates hypoxia

The low oxygen concentrations in hypoxic regions of tumors may not be limiting for OXPHOS (2), and ATP is generated by OXPHOS in tumors even at very low oxygen tensions $(13,43)$. Therefore OXPHOS inhibition could be an effective way to reduce the consumption of oxygen (the terminal electron acceptor in the ETC), and to consequently increase oxygen availability in the tissue. As a result, oxygen could diffuse into initially hypoxic tumor regions, reducing or eradicating tumor hypoxia. Furthermore, this could be a potential strategy for all hypoxic tumors, not simply those in which OXPHOS is upregulated. Studies in 3-D multicellular spheroids indicate that reducing the OCR can alleviate the central region of hypoxia by increasing the availability of free oxygen (44-46). Mathematical modeling suggests that complete inhibition of oxygen consumption is not required for alleviation of tumor hypoxia, and that even a 30\% decrease in consumption would abolish severe hypoxia $(44,45)$.

There are several possible benefits of modifying hypoxia by reducing the OCR compared to other methods of reducing hypoxia. First, targeting OXPHOS appears to reduce the OCR in a wide range of cancer types, suggesting broad applicability for this approach $(40,42,47)$. Second, diffusion of the inhibitor to poorly vascularized hypoxic regions may not be required, as OXPHOS inhibitors acting primarily on the normoxic regions to reduce the OCR may indirectly lead to higher oxygen levels in regions that are chronically hypoxic prior to treatment by allowing molecular oxygen, which very readily diffuses, to reach formerly hypoxic regions. In contrast, nitroimidazoles must reach all hypoxic regions.

\section{OXPHOS inhibitors with therapeutic potential}

\section{OXPHOS inhibitors with a suitable therapeutic index}

Therapeutically viable OXPHOS inhibitors must be efficacious in vitro and in vivo at concentrations that are achievable in the tumors of patients. Drug plasma concentrations determined in previous pharmacokinetic studies for FDA approved drugs may be used as a 
surrogate, although the concentration in the tumors may be lower. Furthermore, the metabolism of the inhibitors, and the effects of any secondary metabolites have to be considered. A partial list of OXPHOS inhibitors that meet these criteria are shown in Table 2 and Fig. 1, and are discussed below.

Epidemiological and retrospective studies have revealed a lower incidence of cancer and better outcomes in diabetics taking the anti-diabetic, metformin, compared to nondiabetics or diabetics taking alternative medications $(3,48)$. In vitro studies have demonstrated that metformin reduces the OCR in many cancer cell lines, a response that is not correlated with its anti-proliferative effect $(40,42,47)$. Many subsequent in vivo studies have revealed that metformin inhibits tumor growth in a variety of different models $(28,29,48,49)$. It also reduces hypoxia in spheroids and xenografted tumors, with a corresponding improvement in radiation sensitivity $(40,42)$. As a consequence of these findings, metformin is already in several hundred ongoing clinical trials to assess its efficacy as an anti-cancer therapeutic. A key mechanism of action of metformin in cancer cells in vitro is complex I inhibition $(48,49)$. This results in a decrease in ATP production and thus activation of AMPK and inhibition of mTORC1 . The growth inhibition of HCT116 xenograft tumors by metformin is complex I dependent, suggesting that complex I inhibition is the mechanism underlying the growth inhibitory effect at least in this model (49). The anti-tumorigenic properties of metformin may also be partly due to systemically lowered insulin levels, resulting in reduced activation of insulin-like receptor tyrosine kinases such as IGF1 in cancer cells (48). However, there is concern that the concentrations of metformin reached in tumors are not sufficient to inhibit complex I. This has led some groups to develop particular mitochondria-targeting metformin analogues with enhanced efficacy in a physiological environment (50), and to study other biguanides with higher potency, such as phenformin. Although phenformin was withdrawn from clinical use in the 1970 s due to a high risk of fatal lactic acidosis, it may have application as an anti-cancer therapeutic at lower doses. Indeed, recent work has shown that 
phenformin causes growth delay of xenograft tumors, and that this is also likely mediated by complex I inhibition $(15,33,35,49,51)$.

Atovaquone is FDA approved to treat pneumocystis pneumonia and malaria, caused by the parasites, Pneumocystis jirovecii and Plasmodium falciparum, respectively (52). It has an excellent safety profile, and has been used in the clinic for over 30 years with approximately 3.7 million prescriptions being issued in the USA every year. It is a ubiquinone analogue that acts as a complex III inhibitor in parasites, cancer cell lines, and breast cancer stem cells, causing a reduction in the OCR and alleviating tumor hypoxia at pharmacologically achievable concentrations (40,53-56). Correspondingly, there is an improvement in radiation response in spheroids and in xenografted tumors following atovaquone treatment (40). Atovaquone also has anti-tumor activity in U266 multiple myeloma xenografts, although this could be due to inhibition of STAT3 rather than complex III (57).

Arsenic trioxide is a complex IV inhibitor that is FDA approved for the treatment of acute promyelocytic leukemia (APL), and is being investigated in other cancer types. It reduces hypoxia in Lewis lung carcinoma (LLC) and transplantable mouse liver (TLT) tumors, leading to an improvement in radiation response (41). Nitric oxide (NO) is a vasodilator, but also inhibits complex IV (58). NO is released from compounds such as isosorbide dinitrate, xanthinol nicotinate and $S$-nitrosocaptopril, and endogenous NO can be stimulated by administration of insulin (39). NO delivered by these methods causes a decrease in tumor hypoxia and corresponding enhancement of radiation response, an effect that may be mediated both by improved blood flow and OXPHOS inhibition (39). Hydrocortisone is another compound that inhibits complex IV in isolated mitochondria, and is able to alleviate hypoxia in TLT and FSaII fibrosarcoma tumors, ameliorating radiation response $(59,60)$. 
There are comparatively few well characterized complex II inhibitors, but lonidamine and the vitamin E analogue, $\alpha$-tocopheryl succinate ( $\alpha$-TOS), may have suitable therapeutic indices. Lonidamine is classically described as an inhibitor of glycolytic hexokinases, but has recently been shown to inhibit complex II in isolated mitochondria and in DB-1 melanoma cells $(5,61)$. Despite early promise, it was not beneficial in two randomized phase III trials in combination with chemotherapy, so is no longer being developed clinically (5). $\alpha$-TOS has not yet been studied clinically, but causes growth reduction in H-Ras transformed Chinese Hamster fibroblast tumors via complex II inhibition, an effect reversed in tumors with dysfunctional complex II and rescued by reconstitution of complex II activity (62).

Aside from the biguanides, several other compounds targeting complex I may have a suitable therapeutic index. Carboxyamidotriazole (CAI) is a putative complex I inhibitor that was initially characterized as an agonist of non-voltage-gated calcium channels and inhibits angiogenesis, tumor growth and metastatic potential $(63,64)$. CAI inhibits growth of a wide range of cell lines in vitro and in vivo, and has an additive effect in Lewis lung carcinoma (LLC) xenografts in combination with the glycolytic inhibitor, 2-deoxyglucose (63). Despite these promising preclinical studies, CAI failed to demonstrate clinical benefits in NSCLC, glioblastoma or metastatic renal cell carcinoma (64). CAI might be more successful if used to treat cancers with upregulated OXPHOS. ME344 is a complex I inhibitor that synergizes with TKIs to induce tumor control in a spontaneous breast cancer model, and is currently being combined with bevacizumab in a clinical trial in early HER2-negative breast cancer patients $(65,66)$. Fenofibrate is a peroxisome proliferator-activated receptor $\alpha$ (PPAR $\alpha)$ agonist approved to treat hyperlipidemia, but also inhibits complex I in isolated mitochondria and in glioblastoma cell lines, causing a significant growth decrease in an orthotopic U87 intracranial glioblastoma model $(67,68)$. However, fenofibrate is hydrolyzed in the blood to fenofibric acid, which does not inhibit complex I, so the effect was only observed following direct intracranial delivery of fenofibrate. Meta-iodobenzylguanidine (mIBG) is a tumor 
targeted radiopharmaceutical that inhibits both complexes I and III, reducing hypoxia in melanoma xenografts $(69,70)$.

In summary, the studies of biguanides and other OXPHOS modulators demonstrate that complex I is a particularly attractive target. Caution is required however, as the novel BAY87-2243 complex I inhibitor alleviated hypoxia and improved radiation response without toxicity in mice, but the initial phase I trial had to be terminated due to unexpected toxicity $(71,72)$. Therefore the pharmacokinetic properties and potency of OXPHOS inhibitors must be carefully tailored.

OXPHOS inhibitors studied in vitro with potential as therapeutics

At first glance it would not appear fruitful to study an OXPHOS inhibitor if the plasma concentration achievable in patients is reported to be lower than the concentration required to cause a significant decrease in the OCR of cancer cells. However, in vivo studies with inhibitors that show promise in vitro may be warranted, as it is possible that higher dose regimens could be effective, that the compound could accumulate in the tumor, or that even a mild reduction in the OCR by these compounds could translate to a significant anti-tumor effect or elevated free oxygen levels. For example, metformin reaches concentrations of up to $184 \mu \mathrm{M}$ in mouse xenograft tumors, which is sufficient to activate AMPK (73). However, a more than 300-fold excess of metformin is required to achieve a comparable effect in vitro, suggesting that the complex metabolic flux of the tumor microenvironment is poorly modeled in vitro (73). It may also be of interest to attempt novel routes of administration or chemical modification of compounds with poor bioavailability in order to improve their bioavailability. Selected examples of OXPHOS inhibitors studied in vitro are shown in in Table 3, but future in vivo experiments are required to determine the suitability of these compounds as anticancer therapeutics. 


\section{Conclusions}

Many recent studies have demonstrated that OXPHOS is upregulated in a variety of cancers, potentially rendering them sensitive to OXPHOS inhibition. Furthermore, OXPHOS inhibition has been shown to reduce the OCR, alleviating tumor hypoxia and even to be effective in some cancers with mtDNA mutations. Repurposing of FDA approved drugs has revealed that many well-tolerated, widely prescribed drugs such as metformin, arsenic trioxide and atovaquone act as OXPHOS inhibitors, and have potential as anti-cancer therapeutics. High-throughput screening approaches could be used to reveal similar compounds with therapeutic potential.

Overall, there is increasing interest in the use of OXPHOS inhibitors against malignant cells, but careful evaluation of potency, pharmacokinetics, and dose regimes will be required, as classical mitochondrial poisons and potent novel inhibitors such as BAY872243 can cause unacceptable side-effects. Indeed, some inhibitors may be best suited to treat cancers in which OXPHOS is upregulated, but may need to be avoided by some patient groups, such as those with pre-existing mitochondrial disorders. Ultimately, clinical trials with clear patient stratification will be required to determine whether OXPHOS inhibitors have a suitable therapeutic index. Although the example of thalidomide proves that drug repurposing can be successful, funding expensive late phase clinical trials for such drugs may be challenging, and pharmaceutical-driven development of novel inhibitors may be required to overcome this issue. There is also potential for synergy of OXPHOS inhibitors with conventional chemotherapeutics, targeted therapies such as Src, EGFR and BRAF inhibitors, vascular modifiers, inhibitors of other metabolic pathways such as glycolysis, and with radiation in hypoxic tumors.

Therefore, cancers intrinsically sensitive to OXPHOS inhibition should continue to be characterized, environmental and epigenetic drivers of cancer cell susceptibility to OXPHOS inhibitors must be fully recognized, and combinations with other therapies explored. 


\section{Conflict of interest}

The authors declare no competing interests.

\section{Acknowledgements}

The funding sources were Cancer Research UK, Medical Research Council, and National Institute for Health Research Biomedical Research Centre, Oxford. G.S.H. is supported by a Cancer Research UK Clinician Scientist Award (Grant number C34326/A13092). We thank James Coates for helpful discussions.

\section{Author contributions}

T.M.A. wrote the manuscript with advice from L.A.K.-S., G.S.H, and W.G.McK. The manuscript outline was conceived by T.M.A., L.A.K.-S. and W.G.McK.

\section{References}

1. Weinberg SE, Chandel NS. Targeting mitochondria metabolism for cancer therapy. Nat Chem Biol 2015;11(1):9-15 doi 10.1038/nchembio.1712.

2. Moreno-Sanchez R, Rodriguez-Enriquez S, Marin-Hernandez A, Saavedra E. Energy metabolism in tumor cells. FEBS J 2007;274(6):1393-418 doi 10.1111/j.1742-4658.2007.05686.x.

3. Koritzinsky M. Metformin: A Novel Biological Modifier of Tumor Response to Radiation Therapy. International journal of radiation oncology, biology, physics 2015;93(2):454-64 doi 10.1016/j.ijrobp.2015.06.003.

4. Wang W, Karamanlidis G, Tian R. Novel targets for mitochondrial medicine. Science translational medicine 2016;8(326):326rv3 doi 10.1126/scitranslmed.aac7410. 
5. Martinez-Outschoorn UE, Peiris-Pages M, Pestell RG, Sotgia F, Lisanti MP. Cancer metabolism: a therapeutic perspective. Nat Rev Clin Oncol 2016 doi 10.1038/nrclinonc.2016.60.

6. Wallace DC. Mitochondria and cancer. Nat Rev Cancer 2012;12(10):685-98 doi 10.1038/nrc3365.

7. Zong WX, Rabinowitz JD, White E. Mitochondria and Cancer. Mol Cell 2016;61(5):667-76 doi 10.1016/j.molcel.2016.02.011.

8. Bost F, Decoux-Poullot AG, Tanti JF, Clavel S. Energy disruptors: rising stars in anticancer therapy? Oncogenesis 2016;5:e188 doi 10.1038/oncsis.2015.46.

9. Viale A, Corti D, Draetta GF. Tumors and mitochondrial respiration: a neglected connection. Cancer Res 2015;75(18):3685-6 doi 10.1158/0008-5472.CAN-150491.

10. Yu M. Generation, function and diagnostic value of mitochondrial DNA copy number alterations in human cancers. Life Sci 2011;89(3-4):65-71 doi 10.1016/j.lfs.2011.05.010.

11. Gaude E, Frezza C. Tissue-specific and convergent metabolic transformation of cancer correlates with metastatic potential and patient survival. Nature communications 2016;7:13041 doi 10.1038/ncomms13041.

12. Cavalli LR, Varella-Garcia M, Liang BC. Diminished tumorigenic phenotype after depletion of mitochondrial DNA. Cell Growth Differ 1997;8(11):1189-98.

13. Weinberg F, Hamanaka R, Wheaton WW, Weinberg S, Joseph J, Lopez M, et al. Mitochondrial metabolism and ROS generation are essential for Kras-mediated tumorigenicity. Proceedings of the National Academy of Sciences of the United States of America 2010;107(19):8788-93 doi 10.1073/pnas.1003428107.

14. Larman TC, DePalma SR, Hadjipanayis AG, Cancer Genome Atlas Research N, Protopopov A, Zhang J, et al. Spectrum of somatic mitochondrial mutations in five 
cancers. Proceedings of the National Academy of Sciences of the United States of America 2012;109(35):14087-91 doi 10.1073/pnas.1211502109.

15. Birsoy K, Possemato R, Lorbeer FK, Bayraktar EC, Thiru P, Yucel B, et al. Metabolic determinants of cancer cell sensitivity to glucose limitation and biguanides. Nature 2014;508(7494):108-12 doi 10.1038/nature13110.

16. Zu XL, Guppy M. Cancer metabolism: facts, fantasy, and fiction. Biochemical and biophysical research communications 2004;313(3):459-65.

17. Vaupel P, Mayer A. Availability, not respiratory capacity governs oxygen consumption of solid tumors. The international journal of biochemistry \& cell biology 2012;44(9):1477-81 doi 10.1016/j.biocel.2012.05.019.

18. Reznik E, Miller ML, Senbabaoglu Y, Riaz N, Sarungbam J, Tickoo SK, et al. Mitochondrial DNA copy number variation across human cancers. eLife 2016;5 doi 10.7554/eLife.10769.

19. Viale A, Pettazzoni P, Lyssiotis CA, Ying H, Sanchez N, Marchesini M, et al. Oncogene ablation-resistant pancreatic cancer cells depend on mitochondrial function. Nature 2014;514(7524):628-32 doi 10.1038/nature13611.

20. Hensley CT, Faubert B, Yuan Q, Lev-Cohain N, Jin E, Kim J, et al. Metabolic Heterogeneity in Human Lung Tumors. Cell 2016;164(4):681-94 doi 10.1016/j.cell.2015.12.034.

21. Davidson SM, Papagiannakopoulos T, Olenchock BA, Heyman JE, Keibler MA, Luengo A, et al. Environment Impacts the Metabolic Dependencies of Ras-Driven Non-Small Cell Lung Cancer. Cell Metab 2016;23(3):517-28 doi 10.1016/j.cmet.2016.01.007.

22. Whitaker-Menezes D, Martinez-Outschoorn UE, Flomenberg N, Birbe RC, Witkiewicz AK, Howell A, et al. Hyperactivation of oxidative mitochondrial metabolism in epithelial cancer cells in situ: visualizing the therapeutic effects of 
metformin in tumor tissue. Cell Cycle 2011;10(23):4047-64 doi 10.4161/cc.10.23.18151.

23. Zacksenhaus E, Shrestha M, Liu JC, Vorobieva I, Chung PED, Ju Y, et al. Mitochondrial OXPHOS Induced by RB1 Deficiency in Breast Cancer: Implications for Anabolic Metabolism, Stemness, and Metastasis. Trends Cancer 2017;3(11):768-79 doi 10.1016/j.trecan.2017.09.002.

24. Jones RA, Robinson TJ, Liu JC, Shrestha M, Voisin V, Ju Y, et al. RB1 deficiency in triple-negative breast cancer induces mitochondrial protein translation. J Clin Invest 2016;126(10):3739-57 doi 10.1172/JCI81568.

25. Birkenmeier K, Drose S, Wittig I, Winkelmann R, Kafer V, Doring C, et al. Hodgkin and Reed-Sternberg cells of classical Hodgkin lymphoma are highly dependent on oxidative phosphorylation. Int J Cancer 2016;138(9):2231-46 doi 10.1002/ijc.29934.

26. Caro P, Kishan AU, Norberg E, Stanley IA, Chapuy B, Ficarro SB, et al. Metabolic signatures uncover distinct targets in molecular subsets of diffuse large B cell lymphoma. Cancer Cell 2012;22(4):547-60 doi 10.1016/j.ccr.2012.08.014.

27. Lagadinou ED, Sach A, Callahan K, Rossi RM, Neering SJ, Minhajuddin M, et al. BCL-2 inhibition targets oxidative phosphorylation and selectively eradicates quiescent human leukemia stem cells. Cell Stem Cell 2013;12(3):329-41 doi 10.1016/j.stem.2012.12.013.

28. Scotland S, Saland E, Skuli N, de Toni F, Boutzen H, Micklow E, et al. Mitochondrial energetic and AKT status mediate metabolic effects and apoptosis of metformin in human leukemic cells. Leukemia 2013;27(11):2129-38 doi 10.1038/leu.2013.107. 
29. Lonardo E, Cioffi M, Sancho P, Sanchez-Ripoll Y, Trabulo SM, Dorado J, et al. Metformin targets the metabolic achilles heel of human pancreatic cancer stem cells. PLoS One 2013;8(10):e76518 doi 10.1371/journal.pone.0076518.

30. Telang S, Lane AN, Nelson KK, Arumugam S, Chesney J. The oncoprotein HRasV12 increases mitochondrial metabolism. Mol Cancer 2007;6:77 doi 10.1186/1476-4598-6-77.

31. Kuntz EM, Baquero P, Michie AM, Dunn K, Tardito S, Holyoake TL, et al. Targeting mitochondrial oxidative phosphorylation eradicates therapy-resistant chronic myeloid leukemia stem cells. Nat Med 2017;23(10):1234-40 doi 10.1038/nm.4399.

32. Haq R, Shoag J, Andreu-Perez P, Yokoyama S, Edelman H, Rowe GC, et al. Oncogenic BRAF regulates oxidative metabolism via PGC1alpha and MITF. Cancer Cell 2013;23(3):302-15 doi 10.1016/j.ccr.2013.02.003.

33. Yuan P, Ito K, Perez-Lorenzo R, Del Guzzo C, Lee JH, Shen CH, et al. Phenformin enhances the therapeutic benefit of BRAF(V600E) inhibition in melanoma. Proceedings of the National Academy of Sciences of the United States of America 2013;110(45):18226-31 doi 10.1073/pnas.1317577110.

34. Vazquez F, Lim JH, Chim H, Bhalla K, Girnun G, Pierce K, et al. PGC1alpha expression defines a subset of human melanoma tumors with increased mitochondrial capacity and resistance to oxidative stress. Cancer Cell 2013;23(3):287-301 doi 10.1016/j.ccr.2012.11.020.

35. Shackelford DB, Abt E, Gerken L, Vasquez DS, Seki A, Leblanc M, et al. LKB1 inactivation dictates therapeutic response of non-small cell lung cancer to the metabolism drug phenformin. Cancer Cell 2013;23(2):143-58 doi 10.1016/j.ccr.2012.12.008. 
36. Dhani N, Fyles A, Hedley D, Milosevic M. The clinical significance of hypoxia in human cancers. Seminars in nuclear medicine 2015;45(2):110-21 doi 10.1053/j.semnuclmed.2014.11.002.

37. Higgins GS, O'Cathail SM, Muschel RJ, McKenna WG. Drug radiotherapy combinations: review of previous failures and reasons for future optimism. Cancer Treat Rev 2015;41(2):105-13 doi 10.1016/j.ctrv.2014.12.012.

38. Gilkes DM, Semenza GL, Wirtz D. Hypoxia and the extracellular matrix: drivers of tumour metastasis. Nat Rev Cancer 2014;14(6):430-9 doi 10.1038/nrc3726.

39. Jordan BF, Sonveaux P. Targeting tumor perfusion and oxygenation to improve the outcome of anticancer therapy. Front Pharmacol 2012;3:94 doi 10.3389/fphar.2012.00094.

40. Ashton TM, Fokas E, Kunz-Schughart LA, Folkes LK, Anbalagan S, Huether M, et al. The anti-malarial atovaquone increases radiosensitivity by alleviating tumour hypoxia. Nature communications 2016;7:12308 doi 10.1038/ncomms12308.

41. Diepart C, Karroum O, Magat J, Feron 0, Verrax J, Calderon PB, et al. Arsenic trioxide treatment decreases the oxygen consumption rate of tumor cells and radiosensitizes solid tumors. Cancer Res 2012;72(2):482-90 doi 10.1158/00085472.CAN-11-1755.

42. Zannella VE, Dal Pra A, Muaddi H, McKee TD, Stapleton S, Sykes J, et al. Reprogramming metabolism with metformin improves tumor oxygenation and radiotherapy response. Clin Cancer Res 2013;19(24):6741-50 doi 10.1158/1078-0432.CCR-13-1787.

43. Rumsey WL, Schlosser C, Nuutinen EM, Robiolio M, Wilson DF. Cellular energetics and the oxygen dependence of respiration in cardiac myocytes isolated from adult rat. J Biol Chem 1990;265(26):15392-402. 
44. Grimes DR, Kelly C, Bloch K, Partridge M. A method for estimating the oxygen consumption rate in multicellular tumour spheroids. J R Soc Interface 2014;11(92):20131124 doi 10.1098/rsif.2013.1124.

45. Kelly CJ, Hussien K, Fokas E, Kannan P, Shipley RJ, Ashton TM, et al. Regulation of O consumption by the PI3K and mTOR pathways contributes to tumor hypoxia. Radiother Oncol 2014 doi 10.1016/j.radonc.2014.02.007.

46. Secomb TW, Hsu R, Ong ET, Gross JF, Dewhirst MW. Analysis of the effects of oxygen supply and demand on hypoxic fraction in tumors. Acta oncologica 1995;34(3):313-6.

47. Chowdhury S, Yung E, Pintilie M, Muaddi H, Chaib S, Yeung M, et al. MATE2 Expression Is Associated with Cancer Cell Response to Metformin. PLoS One 2016;11(12):e0165214 doi 10.1371/journal.pone.0165214.

48. Pernicova I, Korbonits M. Metformin--mode of action and clinical implications for diabetes and cancer. Nat Rev Endocrinol 2014;10(3):143-56 doi 10.1038/nrendo.2013.256.

49. Wheaton WW, Weinberg SE, Hamanaka RB, Soberanes S, Sullivan LB, Anso E, et al. Metformin inhibits mitochondrial complex I of cancer cells to reduce tumorigenesis. eLife 2014;3:e02242 doi 10.7554/eLife.02242.

50. Cheng G, Zielonka J, Ouari O, Lopez M, McAllister D, Boyle K, et al. MitochondriaTargeted Analogues of Metformin Exhibit Enhanced Antiproliferative and Radiosensitizing Effects in Pancreatic Cancer Cells. Cancer Res 2016;76(13):3904-15 doi 10.1158/0008-5472.CAN-15-2534.

51. Appleyard MV, Murray KE, Coates PJ, Wullschleger S, Bray SE, Kernohan NM, et al. Phenformin as prophylaxis and therapy in breast cancer xenografts. Br J Cancer 2012;106(6):1117-22 doi 10.1038/bjc.2012.56. 
52. Nixon GL, Moss DM, Shone AE, Lalloo DG, Fisher N, O'Neill PM, et al. Antimalarial pharmacology and therapeutics of atovaquone. The Journal of antimicrobial chemotherapy 2013;68(5):977-85 doi 10.1093/jac/dks504.

53. Birth D, Kao WC, Hunte C. Structural analysis of atovaquone-inhibited cytochrome bc1 complex reveals the molecular basis of antimalarial drug action. Nature communications 2014;5:4029 doi 10.1038/ncomms5029.

54. Dixon R, Pozniak AL, Watt HM, Rolan P, Posner J. Single-dose and steady-state pharmacokinetics of a novel microfluidized suspension of atovaquone in human immunodeficiency virus-seropositive patients. Antimicrobial agents and chemotherapy 1996;40(3):556-60.

55. Falloon J, Sargent S, Piscitelli SC, Bechtel C, LaFon SW, Sadler B, et al. Atovaquone suspension in HIV-infected volunteers: pharmacokinetics, pharmacodynamics, and TMP-SMX interaction study. Pharmacotherapy 1999;19(9):1050-6.

56. Fiorillo M, Lamb R, Tanowitz HB, Mutti L, Krstic-Demonacos M, Cappello AR, et al. Repurposing atovaquone: Targeting mitochondrial complex III and OXPHOS to eradicate cancer stem cells. Oncotarget 2016 doi 10.18632/oncotarget.9122.

57. Xiang M, Kim H, Ho VT, Walker SR, Bar-Natan M, Anahtar M, et al. Gene expression-based discovery of atovaquone as a STAT3 inhibitor and anti-cancer agent. Blood 2016 doi 10.1182/blood-2015-07-660506.

58. Clementi E, Brown GC, Foxwell N, Moncada S. On the mechanism by which vascular endothelial cells regulate their oxygen consumption. Proceedings of the National Academy of Sciences of the United States of America 1999;96(4):155962.

59. Crokart N, Radermacher K, Jordan BF, Baudelet C, Cron GO, Gregoire V, et al. Tumor radiosensitization by antiinflammatory drugs: evidence for a new 
mechanism involving the oxygen effect. Cancer Res 2005;65(17):7911-6 doi 10.1158/0008-5472.CAN-05-1288.

60. Simon N, Jolliet P, Morin C, Zini R, Urien S, Tillement JP. Glucocorticoids decrease cytochrome c oxidase activity of isolated rat kidney mitochondria. FEBS letters 1998;435(1):25-8.

61. Guo L, Shestov AA, Worth AJ, Nath K, Nelson DS, Leeper DB, et al. Inhibition of Mitochondrial Complex II by the Anticancer Agent Lonidamine. J Biol Chem 2016;291(1):42-57 doi 10.1074/jbc.M115.697516.

62. Dong LF, Freeman R, Liu J, Zobalova R, Marin-Hernandez A, Stantic M, et al. Suppression of tumor growth in vivo by the mitocan alpha-tocopheryl succinate requires respiratory complex II. Clin Cancer Res 2009;15(5):1593-600 doi 10.1158/1078-0432.CCR-08-2439.

63. Ju R, Guo L, Li J, Zhu L, Yu X, Chen C, et al. Carboxyamidotriazole inhibits oxidative phosphorylation in cancer cells and exerts synergistic anti-cancer effect with glycolysis inhibition. Cancer letters 2016;370(2):232-41 doi 10.1016/j.canlet.2015.10.025.

64. Johnson EA, Marks RS, Mandrekar SJ, Hillman SL, Hauge MD, Bauman MD, et al. Phase III randomized, double-blind study of maintenance CAI or placebo in patients with advanced non-small cell lung cancer (NSCLC) after completion of initial therapy (NCCTG 97-24-51). Lung Cancer 2008;60(2):200-7 doi 10.1016/j.lungcan.2007.10.003.

65. Lim SC, Carey KT, McKenzie M. Anti-cancer analogues ME-143 and ME-344 exert toxicity by directly inhibiting mitochondrial NADH: ubiquinone oxidoreductase (Complex I). Am J Cancer Res 2015;5(2):689-701. 
66. Navarro P, Bueno MJ, Zagorac I, Mondejar T, Sanchez J, Mouron S, et al. Targeting Tumor Mitochondrial Metabolism Overcomes Resistance to Antiangiogenics. Cell Rep 2016;15(12):2705-18 doi 10.1016/j.celrep.2016.05.052.

67. Brunmair B, Lest A, Staniek K, Gras F, Scharf N, Roden M, et al. Fenofibrate impairs rat mitochondrial function by inhibition of respiratory complex I. J Pharmacol Exp Ther 2004;311(1):109-14 doi 10.1124/jpet.104.068312.

68. Wilk A, Wyczechowska D, Zapata A, Dean M, Mullinax J, Marrero L, et al. Molecular mechanisms of fenofibrate-induced metabolic catastrophe and glioblastoma cell death. Molecular and cellular biology 2015;35(1):182-98 doi 10.1128/MCB.00562-14.

69. Burd R, Lavorgna SN, Daskalakis C, Wachsberger PR, Wahl ML, Biaglow JE, et al. Tumor oxygenation and acidification are increased in melanoma xenografts after exposure to hyperglycemia and meta-iodo-benzylguanidine. Radiation research 2003;159(3):328-35.

70. Cornelissen J, Wanders RJ, Van Gennip AH, Van den Bogert C, Voute PA, Van Kuilenburg AB. Meta-iodobenzylguanidine inhibits complex I and III of the respiratory chain in the human cell line Molt-4. Biochemical pharmacology 1995;49(4):471-7.

71. Chang E, Liu H, Unterschemmann K, Ellinghaus P, Liu S, Gekeler V, et al. 18F-FAZA PET imaging response tracks the reoxygenation of tumors in mice upon treatment with the mitochondrial complex I inhibitor BAY 87-2243. Clin Cancer Res 2015;21(2):335-46 doi 10.1158/1078-0432.CCR-14-0217.

72. Ellinghaus P, Heisler I, Unterschemmann K, Haerter M, Beck H, Greschat S, et al. BAY 87-2243, a highly potent and selective inhibitor of hypoxia-induced gene activation has antitumor activities by inhibition of mitochondrial complex I. Cancer medicine 2013;2(5):611-24 doi 10.1002/cam4.112. 
73. Dowling RJ, Lam S, Bassi C, Mouaaz S, Aman A, Kiyota T, et al. Metformin Pharmacokinetics in Mouse Tumors: Implications for Human Therapy. Cell Metab 2016;23(4):567-8 doi 10.1016/j.cmet.2016.03.006.

74. Martinez Marignac VL, Smith S, Toban N, Bazile M, Aloyz R. Resistance to Dasatinib in primary chronic lymphocytic leukemia lymphocytes involves AMPKmediated energetic re-programming. Oncotarget 2013;4(12):2550-66 doi 10.18632/oncotarget.1508.

75. De Rosa V, Iommelli F, Monti M, Fonti R, Votta G, Stoppelli MP, et al. Reversal of Warburg Effect and Reactivation of Oxidative Phosphorylation by Differential Inhibition of EGFR Signaling Pathways in Non-Small Cell Lung Cancer. Clin Cancer Res 2015;21(22):5110-20 doi 10.1158/1078-0432.CCR-15-0375.

76. Berruti A, Bitossi R, Gorzegno G, Bottini A, Alquati P, De Matteis A, et al. Time to progression in metastatic breast cancer patients treated with epirubicin is not improved by the addition of either cisplatin or lonidamine: final results of a phase III study with a factorial design. Journal of clinical oncology : official journal of the American Society of Clinical Oncology 2002;20(20):4150-9.

77. Zhang X, Fryknas M, Hernlund E, Fayad W, De Milito A, Olofsson MH, et al. Induction of mitochondrial dysfunction as a strategy for targeting tumour cells in metabolically compromised microenvironments. Nature communications 2014;5:3295 doi 10.1038/ncomms4295.

78. Harada Y, Ishii I, Hatake K, Kasahara T. Pyrvinium pamoate inhibits proliferation of myeloma/erythroleukemia cells by suppressing mitochondrial respiratory complex I and STAT3. Cancer letters 2012;319(1):83-8 doi 10.1016/j.canlet.2011.12.034.

79. Senkowski W, Zhang X, Olofsson MH, Isacson R, Hoglund U, Gustafsson M, et al. Three-Dimensional Cell Culture-Based Screening Identifies the Anthelmintic Drug 
Nitazoxanide as a Candidate for Treatment of Colorectal Cancer. Mol Cancer Ther 2015;14(6):1504-16 doi 10.1158/1535-7163.MCT-14-0792.

80. Villani LA, Smith BK, Marcinko K, Ford RJ, Broadfield LA, Green AE, et al. The diabetes medication Canagliflozin reduces cancer cell proliferation by inhibiting mitochondrial complex-I supported respiration. Mol Metab 2016;5(10):1048-56 doi 10.1016/j.molmet.2016.08.014.

81. Garcia-Ruiz I, Solis-Munoz P, Fernandez-Moreira D, Munoz-Yague T, SolisHerruzo JA. Pioglitazone leads to an inactivation and disassembly of complex I of the mitochondrial respiratory chain. BMC Biol 2013;11:88 doi 10.1186/17417007-11-88.

82. Hanefeld M. Pharmacokinetics and clinical efficacy of pioglitazone. Int J Clin Pract Suppl 2001(121):19-25.

83. Nadanaciva S, Bernal A, Aggeler R, Capaldi R, Will Y. Target identification of drug induced mitochondrial toxicity using immunocapture based OXPHOS activity assays. Toxicol In Vitro 2007;21(5):902-11 doi 10.1016/j.tiv.2007.01.011.

84. Koshkin V, Ailles LE, Liu G, Krylov SN. Metabolic Suppression of a Drug-Resistant Subpopulation in Cancer Spheroid Cells. J Cell Biochem 2016;117(1):59-65 doi $10.1002 / j c b .25247$.

85. Ehrnebo M, Odar-Cederlof I. Binding of amobarbital, pentobarbital and diphenylhydantoin to blood cells and plasma proteins in healthy volunteers and uraemic patients. European journal of clinical pharmacology 1975;8(6):445-53. 


\section{Figures and figure legends}

Fig. 1. Inhibitors of oxidative phosphorylation.

The oxidative phosphorylation (OXPHOS) metabolic pathway generates ATP by transport of electrons to a series of transmembrane protein complexes in the mitochondrial inner membrane, known as the electron transport chain (ETC). The dotted line indicates the flow of electrons through complex I, complex II, Coenzyme Q10 (Q), complex III, cytochrome c (c), and complex IV, with $\mathrm{O}_{2}$ acting as the terminal electron acceptor. Compounds of therapeutic potential being studied as OXPHOS inhibitors in vivo or in the clinic are shown in green, those being studied in vitro are shown in orange, and classical mitochondrial poisons are shown in red. $\alpha \mathrm{TOS}=\alpha$-tocopheryl succinate, $\mathrm{CAI}=$ carboxyamidotriazole, $\mathrm{CO}=$ carbon monoxide, mIBG = meta-iodobenzylguanidine, $\mathrm{MPTP}=1$-methyl 4-phenyl 1,2,3,6 tetrahydropyridine, $\mathrm{NO}=$ nitric oxide.

Fig. 2. The hypothetical effect of OXPHOS inhibition on tumor oxygen tension.

In the absence of OXPHOS inhibition, tumor oxygen tension decreases steadily with increasing distance from tumor vasculature (44). Both tumor areas with limited oxygen diffusion due to the pathological tumor vasculature, and microregions distant from perfused vessels, are therefore chronically hypoxic. Under OXPHOS inhibition, we hypothesize that OXPHOS activity is greatly reduced throughout the tumor, and that the decreased cellular oxygen consumption lowers the slope of the oxygen gradient from the vessels into the tumor tissue. 


\section{Tables}

\begin{tabular}{|c|c|c|c|}
\hline Cancer & Subtype & Associated gene expression & Ref \\
\hline $\begin{array}{l}\text { Acute myeloid leukemia } \\
\text { (AML) }\end{array}$ & AML stem cells & $\uparrow B C L-2$ & $(27)$ \\
\hline $\begin{array}{l}\text { Chronic lymphocytic } \\
\text { leukemia (CLL) }\end{array}$ & $\begin{array}{l}\text { Src-sensitive CLL after Src } \\
\text { inhibition }\end{array}$ & $\downarrow A K T$ & (74) \\
\hline $\begin{array}{l}\text { Classical Hodgkin's } \\
\text { lymphoma }\end{array}$ & & $\uparrow N F \kappa B$ & $(25)$ \\
\hline $\begin{array}{l}\text { Diffuse large B cell } \\
\text { lymphoma (DLBCL) }\end{array}$ & High OXPHOS expression & & $(26)$ \\
\hline Breast & & $\downarrow R B 1$ & $(22,24)$ \\
\hline $\begin{array}{l}\text { Pancreatic ductal } \\
\text { adenocarcinoma (PDAC) }\end{array}$ & PDAC (stem-like) cells & $\uparrow \operatorname{Ras}$ & $(29)$ \\
\hline Lung adenocarcinoma & & & $(18)$ \\
\hline $\begin{array}{l}\text { Non-small cell lung cancer } \\
\text { (NSCLC) }\end{array}$ & $\begin{array}{l}\text { NSCLC after EGFR } \\
\text { inhibition }\end{array}$ & $\downarrow E G F R$ & $(75)$ \\
\hline $\begin{array}{l}\text { Non-small cell lung cancer } \\
\text { (NSCLC) }\end{array}$ & $\begin{array}{l}\text { Oncogenic Kras and loss of } \\
L K B I\end{array}$ & $\downarrow_{L K B 1}$, oncogenic Kras & $(35)$ \\
\hline Endometrial carcinoma & Serous-like endometrial & mtDNA copy number alteration & $(18)$ \\
\hline Melanoma & High OXPHOS expression & $\uparrow P P A R G C 1 A(\mathrm{PGC} 1 \alpha)$ & (34) \\
\hline Melanoma & $\begin{array}{l}\text { BRAF mutant after BRAF } \\
\text { inhibition }\end{array}$ & $\begin{array}{l}\uparrow P P A R G C 1 A(\mathrm{PGC} 1 \alpha), \\
\text { BRAF activating mutation }\end{array}$ & $(32,33)$ \\
\hline Glioma & Low grade glioma & IDH1 activating mutation & $(18)$ \\
\hline $\begin{array}{l}\text { Head and neck, cervix, lung, } \\
\text { brain, bowel, prostate, } \\
\text { pancreas }\end{array}$ & Hypoxic solid tumors & & $(36)$ \\
\hline
\end{tabular}

Table 1 Potential clinical applications of OXPHOS inhibitors 


\begin{tabular}{|c|c|c|c|c|c|}
\hline Compound & Clinical use & Complex & In vivo results & Selected Oncology Clinical Trials & Ref \\
\hline Metformin & Diabetes & I & $\begin{array}{l}\text { Inhibits tumor growth in many tumor types, alleviates } \\
\text { hypoxia and improves ionizing radiation (IR) response }\end{array}$ & Several hundred trials in progress & $(3,42,49)$ \\
\hline Phenformin & Diabetes & I & $\begin{array}{l}\text { Tumor growth delay, NSCLC with oncogenic Kras and } \\
L K B 1 \text { loss, and in MCF7/MDAMB231 }\end{array}$ & Preclinical & $(35,49,51)$ \\
\hline BAY87-2243 & Experimental & I & $\begin{array}{l}\text { Alleviates hypoxia and improves IR response, UT-SCC5 } \\
\text { tumors }\end{array}$ & $\begin{array}{l}\text { Phase I, solid tumors, terminated } \\
\text { NCT01297530, dose escalation }\end{array}$ & $(71,72)$ \\
\hline $\begin{array}{l}\text { Carboxyamido- } \\
\text { triazole }(\mathrm{CAI})\end{array}$ & Experimental & I & Growth delay, LLC tumors & Phase III, NSCLC, completed NCT00003869 & $(63,64)$ \\
\hline ME344 & Experimental & I & Growth delay, PyMT spontaneous breast & $\begin{array}{l}\text { Phase 0, HER2-negative breast, recruiting, } \\
\text { NCT02806817 }\end{array}$ & $(65,66)$ \\
\hline Fenofibrate & Hyperlipidemia & I & Growth delay, U87-MG tumors after intercranial delivery & $\begin{array}{l}\text { Phase II, myeloma, not recruiting, NCT01965834, } \\
\text { dose response }\end{array}$ & $(67,68)$ \\
\hline Lonidamine & Experimental & II & Growth delay in many tumor types & Phase III, breast, completed & $(61,76)$ \\
\hline$\alpha$-TOS & Vitamin E analog & II & Inhibits complex II in Chinese Hamster fibroblast tumors & Preclinical & $(62)$ \\
\hline Atovaquone & Malaria & III & Alleviates hypoxia and improves IR response, $\mathrm{FaDu}$ tumors & $\begin{array}{l}\text { Phase 0, NSCLC, recruiting } \\
\text { NCT02628080, 18F-MISO-PET }\end{array}$ & $(40,56,57)$ \\
\hline Arsenic trioxide & APL & IV & Improves IR response, TLT tumors & Clinical use for APL & $(41,58)$ \\
\hline Hydrocortisone & Eczema & IV & Alleviates hypoxia in FSaII tumors, improves IR response & Preclinical & $(59,60)$ \\
\hline NO & Experimental & IV & Alleviates hypoxia in many tumors, improves IR response & Phase II, NSCLC, not yet recruiting NCT01210378 & $(39)$ \\
\hline mIBG & Radioactive tracer & I \& III & Alleviates hypoxia in melanoma, improves IR response & Preclinical & $(69,70)$ \\
\hline VLX600 & Experimental & I, II \& IV & Growth delay, HT29 tumors & Phase I, solid tumors, recruiting & $(77)$ \\
\hline
\end{tabular}

Table 2 A non-exhaustive list of OXPHOS inhibitors under study in vivo or in the clinic as anti-cancer therapeutics 


\begin{tabular}{|c|c|c|c|c|c|}
\hline Compound & Primary clinical use & $\begin{array}{l}\text { Target } \\
\text { Complex }\end{array}$ & In vitro results & Plasma concentration in patients & Ref \\
\hline Pyrvinium & Anti-helminth & $\mathrm{I}$ & Reduces OCR and spheroid hypoxia at $1 \mu \mathrm{M}$ & $\begin{array}{l}\text { Poor bioavailability but has been } \\
\text { safely delivered i.p. in mice }\end{array}$ & $(78,79)$ \\
\hline Canagliflozin & Anti-diabetic & $\mathrm{I}$ & $\begin{array}{l}\text { Reduces OCR and clonogenic survival at } 10-30 \mu \mathrm{M} \text { in } \\
\text { cancer cell lines }\end{array}$ & $5-30 \mu \mathrm{M}, 50-300 \mathrm{mg} / \mathrm{day}$ & $(80)$ \\
\hline Pioglitazone & Anti-diabetic & I & Complex I inhibition in liver of Pioglitazone treated mice & $4.5 \mu \mathrm{M}, 15-30 \mathrm{mg} / \mathrm{day}$ & $(81,82)$ \\
\hline Rosiglitazone & Anti-diabetic & I & Inhibits at $100 \mu \mathrm{M}$ in isolated mitochondria & $1.04 \mu \mathrm{M}, 4-8 \mathrm{mg} / \mathrm{day}$ & $(83)$ \\
\hline Amobarbital & Sedative & $\mathrm{I}$ & $\begin{array}{l}\text { Suppresses drug-resistant cancer spheroid subpopulation at } \\
1 \mathrm{mM}\end{array}$ & $17.7 \mu \mathrm{M}, 200 \mathrm{mg} /$ day & $(84,85)$ \\
\hline Nefazodone & Anti-depressant & I & $4 \mu \mathrm{M} \mathrm{IC}{ }_{50}$ in isolated mitochondria & $0.92 \mu \mathrm{M}, 100 \mathrm{mg}$ twice/day & $(83)$ \\
\hline Simvastin & Anti-lipidemic & II & $30 \mu \mathrm{M} \mathrm{IC}{ }_{50}$ in isolated mitochondria & $0.02 \mu \mathrm{M}, 5-40 \mathrm{mg} / \mathrm{day}$ & $(83)$ \\
\hline Paroxetine & Anti-depressant & $\mathrm{V}$ & $1.6 \mu \mathrm{M} \mathrm{IC}{ }_{50}$ in isolated mitochondria & $0.06 \mu \mathrm{M}, 10-60 \mathrm{mg} / \mathrm{day}$ & $(83)$ \\
\hline Chlorpromazine & Anti-psychotic & $\mathrm{V}$ & $26 \mu \mathrm{M} \mathrm{IC}_{50}$ in isolated mitochondria & $0.9 \mu \mathrm{M}, 25-75 \mathrm{mg} /$ day & $(83)$ \\
\hline Tamoxifen & Anti-cancer & III-V & $8.8-26.6 \mu \mathrm{M} \mathrm{IC}_{50}$ in isolated mitochondria & $0.16 \mu \mathrm{M}, 20-40 \mathrm{mg} / \mathrm{day}$ & $(83)$ \\
\hline
\end{tabular}

Table 3 OXPHOS Inhibitors studied in vitro with potential as anti-cancer therapeutics 
Figure 1:

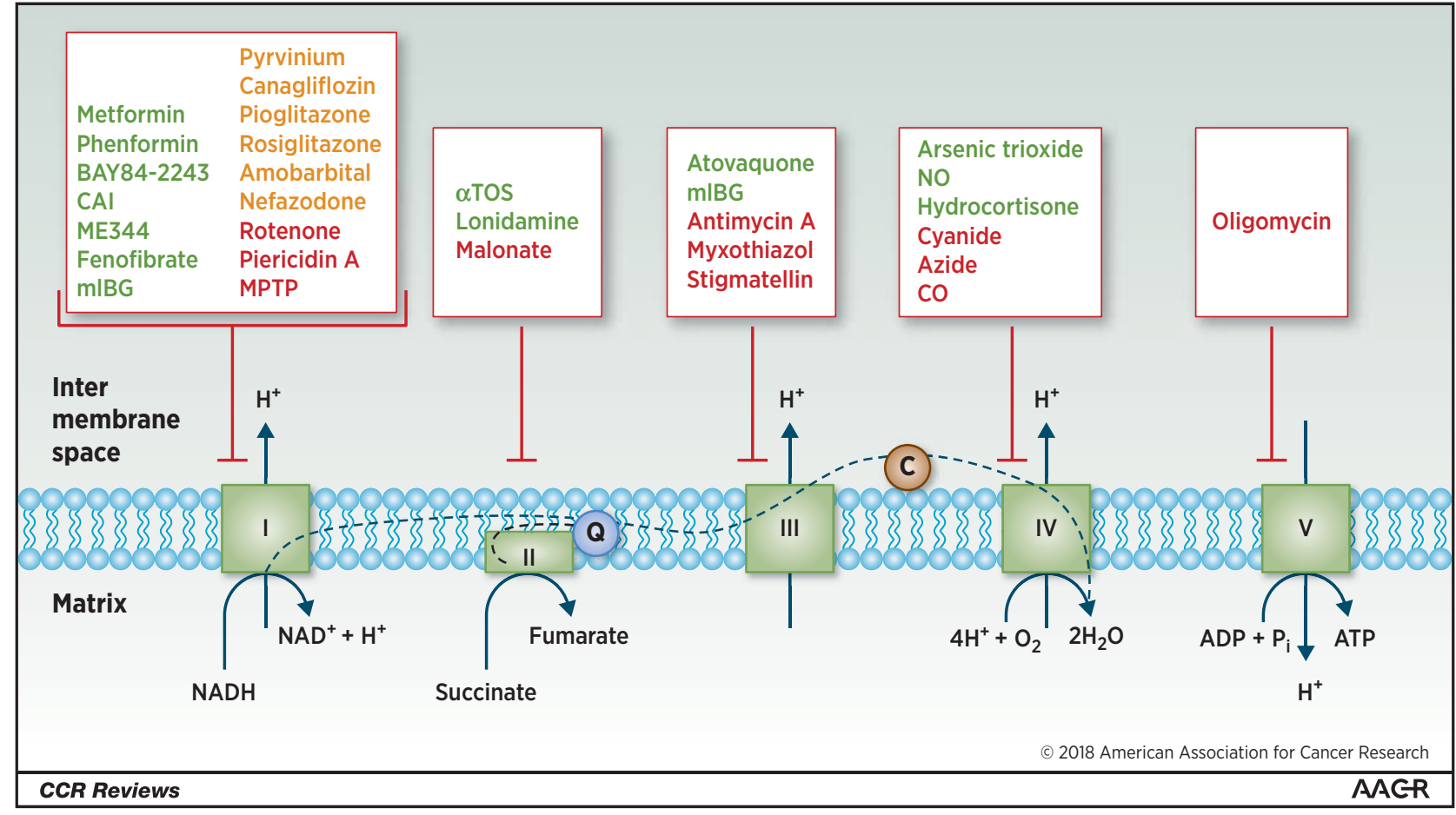


Figure 2:

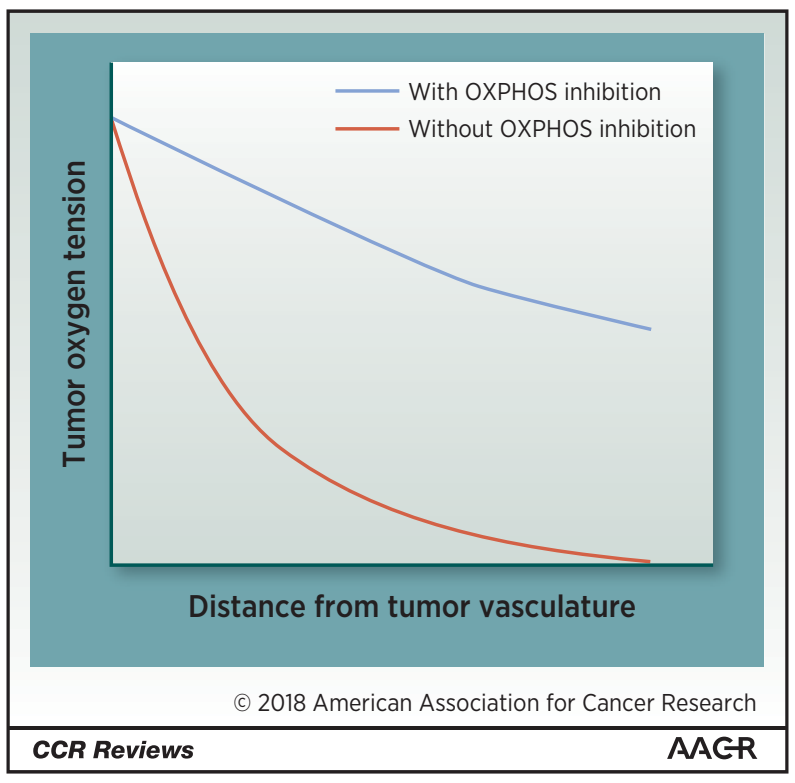

